\title{
Safer Solvent Blends for Food, Dye, and Environmental Analyses Using Reversed-Phase High Performance Liquid Chromatography
}

\author{
Melisa Nallar ${ }^{1} \cdot$ Nicholas Tenaglia $^{1} \cdot$ Gregory Morose $^{2} \cdot$ Hsi-Wu Wong $^{1}$
}

Received: 14 April 2021 / Revised: 5 June 2021 / Accepted: 9 June 2021 / Published online: 18 June 2021

(c) The Author(s) 2021

\begin{abstract}
Liquid chromatography (LC) is a technique widely used to identify and quantify organic compounds in a complex mixture. Typical operations of high-performance liquid chromatography (HPLC) involve continuous use of harmful solvents. Replacing these harmful solvents with safer alternatives will provide significant environmental, health, and safety benefits. In this work, a systematic approach for searching safer solvent blends to replace acetonitrile for reversed-phase (RP) HPLC operations is presented. GreenScreen ${ }^{\circledR}$ for Safer Chemicals was used as the first filter to down-select safer solvent candidates from thousands of chemicals based on their safety ratings. A list of LC operation parameters was then employed to determine final solvent candidates. Finally, Hansen Solubility Parameters in Practice (HSPiP) software was utilized to identify the most probable compositions of blends from these solvents for actual LC testing. It was found that a blend of $75 \%$ ethanol and $25 \%$ methyl acetate by volume provided the chromatograms with the best performance, which had similar response factors and column efficiency compared to acetonitrile when surrogate food additives, dyes, and water pollutants were tested, suggesting that this solvent blend is a potential safer alternative to replace acetonitrile for certain LC applications.
\end{abstract}

Keywords Liquid chromatography $\cdot$ Safer solvents $\cdot$ Acetonitrile $\cdot$ Hansen solubility parameters in practice

\section{Introduction}

High-performance liquid chromatography (HPLC) is a form of column chromatography used to separate, identify, and quantify non-volatile molecules in a complex mixture. This technique is widely used in the manufacturing, pharmaceutical, medical, or even legal industries, consuming about 34 million liters of solvents every year [1]. The reversedphase (RP) HPLC is the most common method of operation, which employs a mixture of two solvents as the mobile phase: water and an organic solvent. The most frequently used organic solvents are methanol, acetonitrile, and tetrahydrofuran [2], all of which pose environmental, health, and safety concerns [3].

Since waste solvents from HPLC applications contain the many compounds in the analyte samples, recycling the

Hsi-Wu Wong

hsiwu_wong@uml.edu

1 Department of Chemical Engineering, University of Massachusetts Lowell, Lowell, MA 01854, USA

2 Massachusetts Toxics Use Reduction Institute, Lowell, MA 01854, USA solvents is impractical due to difficulties in removing or separating these compounds and tight tolerances of contamination for LC operations, making solvent disposal a common practice. Replacing these solvents with safer alternatives has been identified as the most probable solution to address their environmental, health, and safety concerns [4-9]. Historically, identification of solvents for LC applications is through the Snyder Solvent Triangle [10, 11], which classifies 82 common solvents into 8 groups based on Lewis acidity, basicity, and dipolar interactions. The general approach is to find the replacements from the solvents within the same groups. However, the Snyder Triangle only includes the most common 82 solvents. The potential environmental, health, and safety impacts of the solvents are also not explicitly considered so that many safer alternatives are omitted. To enable the selection of the safer alternatives to conventional solvents, several general solvent selection guides exist [12-19], as reviewed by Tobiszewski et al. [20]. This review [20] further proposed a more quantitative and informative approach to screen more than 150 solvents, grouping them into three clusters and ranking them based on their toxicological and hazardous data. An improved solvent assessment procedure was later proposed by the same 
group by including the environmental emission risk of the solvents [21].

Although the solvent selection guides in the literature provide promise in selecting safer solvents, they were not typically developed specifically for LC applications. The possibilities of using blends of two or more solvents are also rarely explored. Solvent blends may possess new solvent properties that cannot be achieved by single components due to nonlinear behaviors created by mixing [22]. To overcome these limitations, Hansen Solubility Parameters in Practice (HSPiP), a powerful software tool that can provide fast estimates of solvent properties [23], particularly for solvent blends, was used in this work to explore possible safer solvent alternatives to acetonitrile for LC applications. The HSPiP database has approximately 10,000 solvents, as opposed to approximately 82 for the Snyder Triangle and hundreds in typical solvent selection guides. It also has the capability to estimate properties of arbitrary blends of the solvents in the database. For these reasons, HSPiP is an ideal approach to discover new safer solvent formulations, particularly solvent blends that were not previously explored for LC operations. In this work, a list of candidate solvents was first obtained by a set of critical safety and LC operation requirements, such as toxicity, water miscibility, UV absorbance, viscosity, cost, and corrosiveness. Solvent properties estimated by HSPiP were then used to identify the possible safer solvent blends for LC operations. The final candidate solvent blends were tested on several common RP-HPLC operations characterizing surrogate food additives, dyes, and wastewater pollutants, following the recommended testing methods for each set of analytes [24-26]. The testing results for using these solvent blends against acetonitrile on RPHPLC are compared and discussed.

\section{Solvent Selection Methodology}

Our solvent selection procedure can be described in the flow chart in Fig. 1. A short list of solvent candidates was first selected according to GreenScreen ${ }^{\circledR}$ for Safer Chemicals benchmarking system $[27,28]$ to have acceptable safety, environmental, and health impacts. The candidate solvents were further down-selected based on LC operation parameters, including UV cutoff, cost, corrosiveness, viscosity, and water miscibility. The final list of safer solvent candidates was then input into HSPiP to determine the most probable solvent blends for actual LC testing.

\section{Solvent Safety Concerns}

GreenScreen ${ }^{\circledR}$ for Safer Chemicals [27, 28], developed by the nonprofit organization Clean Production Action as

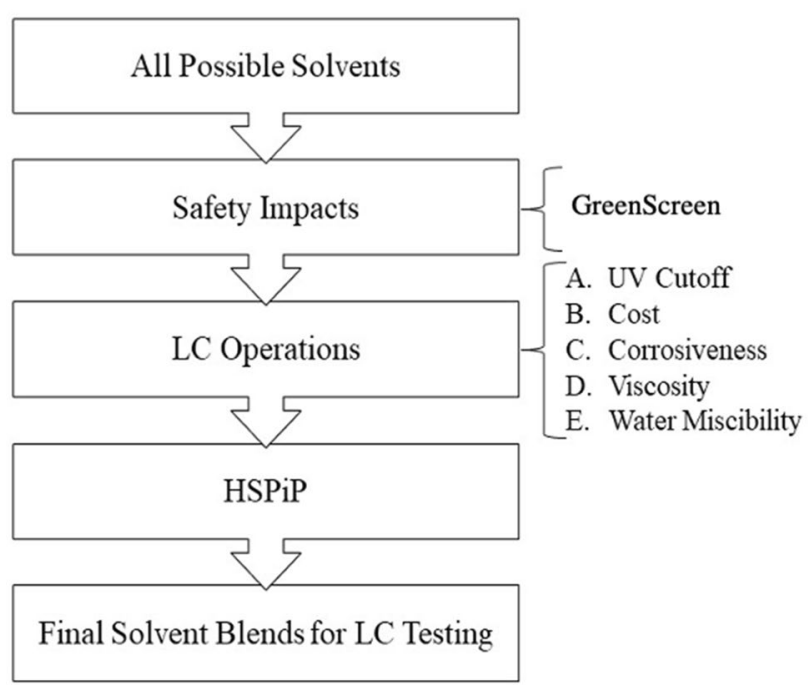

Fig. 1 The selection procedure to down-select an initial list of safer solvent blends for LC testing

a comparative chemical hazard assessment method, was used as the first filter for down-selecting our solvent candidates. In this method, a total of 20 human health, environmental toxicity, fate, and physical hazard endpoints, grouped into five major categories, are used to assess the potential hazard of each chemical (Table A.1). The GreenScreen method ranks the safety of chemicals on a 4-level hazardous scale from the highest to the lowest concerns with benchmark scores of "1" to "4" (Fig. A.1). For chemicals that have not yet had a full GreenScreen evaluation, a GreenScreen score is estimated based upon the presence of chemicals on various authoritative or restricted chemical lists. Since acetonitrile has a score of "1", any solvent with an actual or estimated GreenScreen benchmark at the same level was eliminated from further consideration as a safer alternative.

The GreenScreen method was selected for the screening process in our work for two major reasons. First, it provides a single score for each chemical, which is much more straightforward to use without considering the complex interactions, degree of severity, and tradeoffs between multiple hazard parameters, such as the ones used in the GlaxoSmithKline solvent selection guide [12, 15, 18]. Second, scores of hundreds of different solvents are available in the database, allowing us to explore the possibility of uncommon solvents that were not previously considered, an advantage that is not offered by most alternate singlescore methods, such as the CHEM21 selection guide (which ranks 53 common solvents and 22 less common solvents, although the methodology can be extended to any solvent given sufficient physical data and hazardous statements looked up by the users) [19]. 


\section{LC Operation Requirements}

Once a list of safer solvent candidates was selected by GreenScreen, several critical criteria specifically related to LC operations were then used to determine final solvent candidates to form blends for LC testing. Each chemical was checked to ensure that it has the following properties: UV cutoff lower than $300 \mathrm{~nm}$, cost lower than that of acetonitrile (based on Molbase [29] values), noncorrosive, viscosity lower than or close to $1 \mathrm{cP}$, and miscible with water. For instance, acetic acid, 1-propanol, and cyclopentane have acceptable environmental and health ratings but were rejected due to high corrosiveness, high viscosity, and low water miscibility, respectively. Dimethyl sulfoxide (DMSO), although with a high viscosity of $2.24 \mathrm{cP}$, was included as an exception due to its previous success in replacing methylene chloride for paint stripping products [30], with the hope that it can be blended with another solvent to achieve acceptable viscosity (more discussions later). In addition to the literature data, actual UV tests were also conducted using a UV-visible spectrophotometer (Thermo Scientific, Evaluation 220). The highest UV-cutoff of these candidate solvents were methyl acetate and dimethyl sulfoxide at approximately $250 \mathrm{~nm}$ and $260 \mathrm{~nm}$, respectively (Fig. A.2). Table 1 shows the final list of five solvent candidates selected for subsequent HSPiP evaluation following this procedure. The results for each hazard endpoint from the GreenScreen hazard assessments for the four safer solvents are also included in Table A.2, except for dimethoxymethane which only has an estimated score based upon authoritative chemical lists.

\section{Hansen Solubility Parameters in Practice (HSPiP)}

The final five safer solvent candidates listed in Table 1 were subsequently used in the HSPiP software [35] to discover the best solvent blends among them. HSPiP utilizes the Hansen Solubility Parameter (HSP) theory, which expresses each solvent by three parameters based upon intermolecular forces: dispersion energy $(\delta \mathrm{D})$, polar energy $(\delta \mathrm{P})$, and hydrogen bonding energy $(\delta \mathrm{H})[35]$. Each compound (i.e., solute or analyte), or any arbitrary hypothetical solvent blends, can be expressed as a dot in a three-dimensional space of these parameters. Closer dots represent solvents/ solutes with more similar solubility and that are more miscible. Therefore, the intent is to identify solvent blends with the smallest HSP distance to the target HSP values for food additives and dye compounds.

In this work, the HSP values for a set of surrogate food additives [24] and dye molecules [25] were first looked up from the database in HSPiP. The HSP values for the surrogate food additives were averaged to arrive at a set of target HSP values of $\delta \mathrm{D}=20.0, \delta \mathrm{P}=9.5$, and $\delta \mathrm{H}=14.0$. The HSP values for the surrogate dyes were averaged to

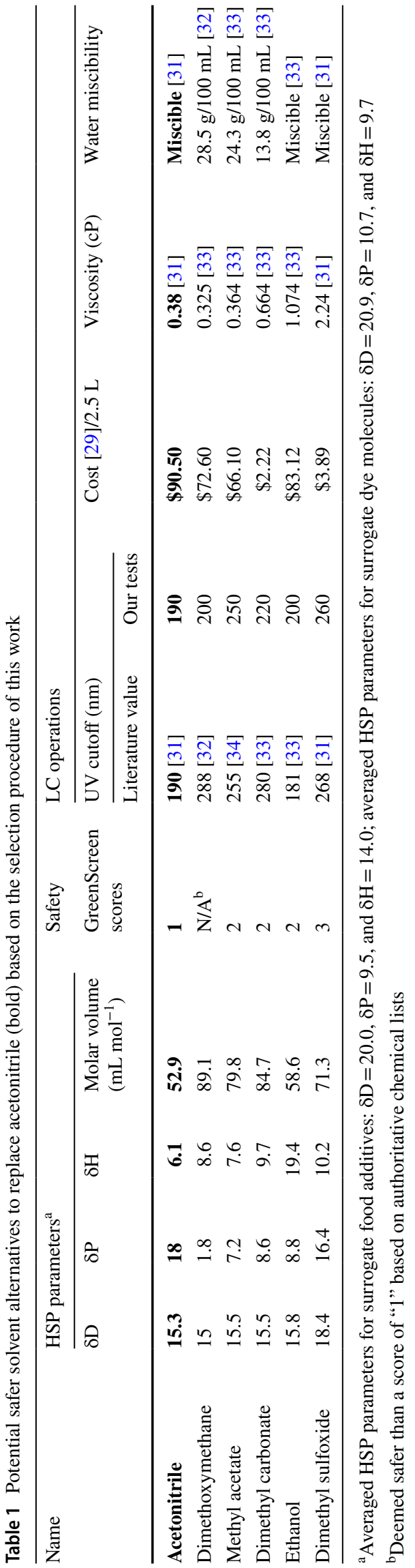


arrive at another set of target HSP values of $\delta \mathrm{D}=20.9$, $\delta \mathrm{P}=10.7$, and $\delta \mathrm{H}=9.7$. The two distinct sets of target HSP values were then used to identify the compositions of the best solvent blends that can be produced from the five solvent candidates in Table 1 for subsequent HPLC testing.

Note that the use of the HSP theory also recognizes the fact that the solvent molar volume has a major effect on solubility performance. In general, the smaller the molar volume, the better the solubility performance. Acetonitrile has a relatively low molar volume of $52.9 \mathrm{~mL} \mathrm{~mol}^{-1}$ (Table 1), resulting in good solubility performance, even though its HSP distances to the target HSP values for food additives and dyes are relatively large compared to the five alternative solvents identified. Among the five alternative solvents, ethanol has a molar volume closest to acetonitrile at $58.6 \mathrm{~mL} \mathrm{~mol}^{-1}$. The other four solvents have molar volumes between 71.3 and $89.1 \mathrm{~mL} \mathrm{~mol}^{-1}$ (Table 1). As a result, it is expected that a tradeoff between the HSP distances to the target HSP values and the solvent molar volumes needs to be considered while formulating the best blends of the alternative solvents for HPLC operations.

By comparing the HSP values of the five safer solvent candidates to the target HSP values for the food additives and dyes, we discovered several challenges using the HSP method to identify the best alternative solvent blends for replacing acetonitrile:

(1) There were two different sets of HSP target values, one for food additives and one for dyes.

(2) Only a limited number of solvents (five) passed all the previous screening criteria such as safety, miscibility, and UV cutoff, limiting the number of solvent combinations and blends that can be explored.

(3) Except for dimethyl sulfoxide (DMSO), four out of the five alternative solvent candidates had HSP dispersion force values $(\delta \mathrm{D})$ between 15.0 and 15.8, posing a challenge to get close to the target HSP dispersion force values for food additives (20.0) and dyes (20.9). Although DMSO has a more preferred $\delta \mathrm{D}$ value of 18.4 , it has a high viscosity of $2.24 \mathrm{cp}$, which could result in high operation pressure of HPLC.

(4) Since viscosity of a solvent blend is typically nonlinear, additional testing was required for the solvent blends involving DMSO which further reduced the amount of potential solvent blend alternatives.

Among the three intermolecular forces used in the HSP theory, hydrogen bonding is in general the strongest and has the greatest impact on solvation. As a result, the solvent molar volume and the hydrogen bonding forces were used as the two determining factors in selecting our final list of alternative solvent blends.
To examine if a different method can help overcome the challenges described above, the approach to determine solute descriptors from chromatographic measurements, developed by Abraham et al. [36], was also visited. This theory includes the following five parameters that can be applied to any molecule: (1) excess molar refraction (denoted as E), (2) dipolarity/polarizability (denoted as S), (3) hydrogen bonding acidity (denoted as A), (4) hydrogen bonding basicity (denoted as B), and (5) the McGowan volume (denoted as V). According to Abbott [37], there is a direct relationship between the Hansen solubility parameters and the Abraham parameters. For instance, the Abraham dipolarity/polarizability parameter $(\mathrm{S})$ corresponds to the polar force $(\delta \mathrm{P})$ of HSP, the Abraham hydrogen bonding parameters (A and B) combined corresponds to the hydrogen bonding force $(\delta \mathrm{H})$ of HSP, and the Abraham excess molar refraction parameter (E) corresponds roughly to the dispersion force ( $\delta \mathrm{D})$ of HSP. Although molar volume is not explicitly a HSP parameter, it is acknowledged as a major factor that affects solubility performance when using the Hansen solubility parameter approach, which should correspond to the Abraham McGowan volume. A comparison of the Hanson solubility parameters and the Abraham parameters for acetonitrile and the five safer solvents considered was conducted in our work. As shown in Table A.3, there is a very strong correlation for molar volume considerations, a strong correlation for hydrogen bond force considerations, and a strong association for polar force considerations between the HSP and the Abraham approaches. The dispersion force was found to have a weak association; however, this is the weakest of the intermolecular forces in the HSP theory and only plays a minor role in the solubility performance. The strong associations between the two approaches suggest that the same selection of solvent blends would result.

\section{Viscosity Tests for Solvent Blends Involving DMSO}

Since DMSO is the alternative solvent candidate that has the HSP dispersion $(\delta \mathrm{D})$ force value closet to the surrogate food additive and dye molecules, the possibility of using DMSO in HPLC was firstly examined. Unfortunately, the major limitation of using DMSO in HPLC is its high viscosity, at approximately $2.24 \mathrm{cP}$, which could lead to high LC operation pressure. To overcome this limitation, mixtures of DMSO with another solvent with low viscosity, such as dimethoxymethane $(0.325 \mathrm{cP})$ or methyl acetate $(0.364 \mathrm{cP})$, were tested at different ratios to create solvent blends that could retain the solubility benefits of DMSO with the viscosity challenge mitigated (Table A.4). These tests were critical because viscosity of a solvent blend is typically nonlinear. Viscosity values of the solvent blends involving DMSO were measured using a vibro viscometer (A\&D, SV-10A, $\mathrm{S} / \mathrm{N}$ T0200682). Each blend was tested three times, and 
average viscosity, along with the standard deviation, is also reported in Table A.4. It was found that a volumetric blend of $75 \%$ DMSO and $25 \%$ methyl acetate has the lowest viscosity compared to the other two blend candidates involving DMSO (Table A.4).

To further investigate the effect of mobile phase viscosity on LC operation pressure for the $75 \% \mathrm{DMSO} / 25 \%$ methyl acetate blend, isocratic runs were conducted with mixing this blend with water at different ratios. It was found that the highest operation pressure of 345 bar occurred at a volumetric ratio of approximately $70 \%$ organic phase $/ 30 \%$ aqueous phase (Table A.5). Note that these isocratic runs were performed at a lower flow rate at $0.8 \mathrm{~mL} \mathrm{~min}^{-1}$ than the recommended $1.08 \mathrm{~mL} \mathrm{~min}^{-1}$ to protect our instrument. Although this pressure value is higher than the highest operation pressure of 160 bar using acetonitrile, it is still lower than the recommended instrument limitation of $400 \mathrm{bar}$ and is safe to operate.

\section{Final Solvent Blends Selected for HPLC Testing}

As described earlier, hydrogen bonding is in general the strongest among the three intermolecular forces used in the HSP theory and has the greatest impact on solvation. In addition, the use of the HSP theory also recognizes the fact that the solvent molar volume has a major effect on solubility performance. As a result, these two parameters were used as the primary factors in selecting our final list of alternative solvent blends.

For solvent blends containing DMSO, a volumetric blend of $75 \%$ DMSO and $25 \%$ methyl acetate was selected for further HPLC testing due to its acceptable viscosity and hydrogen bonding value of 9.6, which is close to the hydrogen bonding value of 9.7 for dyes. The other two selected solvent blends were volumetric blend of $75 \%$ ethanol and
$25 \%$ methyl acetate and volumetric blend of $80 \%$ ethanol and $20 \%$ dimethyl carbonate, because they had hydrogen bonding values of 16.5 and 17.5 , respectively, which is close to the hydrogen bonding value of 14 for the food additives. In addition, the low molar volume of ethanol at $58.6 \mathrm{~mL} \mathrm{~mol}^{-1}$ is also expected to provide better solubility performance. The three selected alternatives are highlighted in bold in Table 2, along with their HSP parameters and molar volume. Their analytical performance on the surrogate food additives, dyes, and wastewater pollutants against that of acetonitrile was studied. Since aqueous phase used in LC requires solvents to be miscible with water, water miscibility of all three solvent blends in all proportions was confirmed by mixing them with water at volumetric ratios of 100:0, 90:10, 60:40, 50:50, 40:60, and 10:90.

\section{HPLC Testing of the Candidate Safer Solvent Blends}

\section{Materials}

Acetonitrile (Fisher Chemical, HPLC grade), dimethyl sulfoxide (methyl sulfoxide, Acros Organics, pure, 99.7\%), ethanol (reagent alcohol, Fisher Chemical, HPLC grade), methyl acetate (Alfa Aesar, 99\%), dimethoxymethane (Acros Organics $99.5+\%$ ), and dimethyl carbonate (Acros Organics, 99\%) were purchased from chemical vendors. Surrogate chemicals used for food analysis include: daidzin (TSZCHEM, 99+\%), glycitin (TSZCHEM, 99+\%), genistin (TSZCHEM, 99+\%), daidzein (4',7-dihydroxyisoflavone, Alfa Aeasar, 98+\%), glycitein (TSZCHEM, 99+\%), genistein (TCI, $98+\%$ ), and apigenin (Tocris Bioscience, for research use only). Formic acid (Acros Organics, 99\%) was used as a buffer to the aqueous mobile phase in the

Table 2 The most promising compositions of solvent blends (by volume fraction) identified by HSPiP

\begin{tabular}{|c|c|c|c|c|c|c|c|c|c|}
\hline \multirow[t]{2}{*}{ Solvent 1} & \multirow[t]{2}{*}{ Vol\% 1} & \multirow[t]{2}{*}{ Solvent 2} & \multirow[t]{2}{*}{ Vol $\% 2$} & \multicolumn{6}{|c|}{ HSP Parameters } \\
\hline & & & & $\delta \mathrm{D}$ & $\delta \mathrm{P}$ & $\delta \mathrm{H}$ & $\begin{array}{l}\text { Molar volume } \\
\left(\mathrm{mL} \mathrm{mol}^{-1}\right)\end{array}$ & $\begin{array}{l}\text { Distance to food } \\
\text { additive target* }\end{array}$ & $\begin{array}{l}\text { Distance to } \\
\text { dye target* }\end{array}$ \\
\hline DMSO & 75 & Methyl acetate & 25 & 17.7 & 14.1 & 9.6 & 73.4 & 7.9 & 7.3 \\
\hline DMSO & 83 & Dimethoxymethane & 17 & 17.8 & 13.9 & 9.9 & 74.3 & 7.5 & 7.0 \\
\hline DMSO & 74 & Dimethyl carbonate & 26 & 17.6 & 14.4 & 10.1 & 74.8 & 7.9 & 7.6 \\
\hline Ethanol & 75 & Methyl acetate & 25 & 15.7 & 8.4 & 16.5 & 63.9 & 9.0 & 12.6 \\
\hline Ethanol & 80 & Dimethyl carbonate & 20 & 15.7 & 8.8 & $\mathbf{1 7 . 5}$ & 63.8 & 9.3 & 13.1 \\
\hline \multicolumn{4}{|c|}{ Acetonitrile } & 15.3 & 18 & 6.1 & 52.9 & 14.9 & 13.9 \\
\hline \multicolumn{4}{|c|}{ Mean HSP values for the surrogate food additives } & 20.0 & 9.5 & 14 & & & \\
\hline \multicolumn{4}{|c|}{ Mean HSP values for the surrogate dyes } & 20.9 & 10.7 & 9.7 & & & \\
\hline
\end{tabular}

The three blends highlighted in bold were selected for actual HPLC testing

*The distance indicates how close the candidate solvent blend is to the target values 
food analysis method. Surrogate chemicals used for dye analysis include: disperse Blue 1 (Sigma-Aldrich, 30\%), disperse Blue 3 (Sigma-Aldrich, 20\%), disperse Blue 106 (Dr. Ehrenstorfer, 99\%), disperse Yellow 3 (Sigma-Aldrich, HPLC grade, $96+\%$ ), disperse Orange 3 (Sigma-Aldrich, HPLC grade, $96+\%$ ), disperse Red 1 (Sigma-Aldrich, $96+\%$ ), disperse Blue 35 (Sigma-Aldrich, for microscopy), disperse Blue 124 (Sigma-Aldrich, for microscopy), and disperse Orange 37 (Sigma-Aldrich, HPLC grade, 96+\%). Methanol (Alfa Aesar, environmental grade, 99.8+\%) was used during the preparation of the standard solutions of dye analytes. In dye analysis, ammonium acetate (Fisher Chemical, LC/MS grade) and formic acid (Fisher Chemical, LC/ MS grade) were added to the aqueous mobile phase to adjust the $\mathrm{pH}$. Surrogate wastewater pollutants include: atenolol (Tocris Bioscience, for research use only), sucralose (TCI, $98+\%$ ) sulfamethoxazole (MP Biomedicals, for research use only), tris(chloro-isopropyl)phosphate (TCPP, mixture of isomers, analytical standard), and Triclosan (EMD Millipore, for research use only).

\section{Preparation of Standard Solutions}

The standard solutions for food additives were prepared following the USP isoflavone method [38]. Sample solutions were diluted with an acetonitrile-water mixture ( $2: 3$ by volume). The concentrations of the analytes were prepared between 10 and $120 \mu \mathrm{g} \mathrm{mL}^{-1}$. The standard solutions for dye molecules were dissolved in methanol and sequentially diluted with an acetonitrile-water mixture ( $2: 3$ by volume). Deionized water was used for the preparation of standard solutions for wastewater analytes. To dissolve triclosan, ethanol was also added at approximately $0.1 \%$ by volume.

\section{HPLC Testing}

The actual testing of solvent blends was conducted on an Agilent 1100 HPLC equipped with a variable wavelength detector (VWD). The recommended standard methods for the sets of surrogate compounds were followed [24-26]. Specifically, for food additive analysis, a Waters CORTECS C18 column with an inner diameter of $3.0 \mathrm{~mm}$, a length of $100 \mathrm{~mm}$, and a packing particle size of $2.7 \mu \mathrm{m}$ was used. In the aqueous mobile phase, $0.1 \mathrm{vol} \%$ of formic acid was added as a buffer. The injection volume for each analysis was $2 \mu \mathrm{L}$. For dye analysis, a Waters XBridge C18 column with an inner diameter of $2.1 \mathrm{~mm}$, a length of $150 \mathrm{~mm}$, and a packing particle size of $5.0 \mu \mathrm{m}$ was used. $10 \mathrm{mmol}$ of ammonium acetate was added as a buffer in the aqueous mobile phase. The injection volume for each analysis was $5 \mu \mathrm{L}$. For water pollutant analysis, a Waters Symmetry C8 with an inner diameter of $2.1 \mathrm{~mm}$, a length of $100 \mathrm{~mm}$, and a packing particle size of $3.5 \mu \mathrm{m}$ was used. $0.1 \mathrm{vol} \%$ of formic acid was added as a buffer in the aqueous mobile phase. The injection volume for each analysis was $80 \mu \mathrm{L}$. For all runs, the column temperature was kept constant at $30^{\circ} \mathrm{C}$ and the wavelength of the VWD was set at $260 \mathrm{~nm}$. To clearly separate all peaks representing the surrogate compounds, the gradient elution recommended in each standard method for acetonitrile was slightly modified for each safer solvent blend tested (see Tables A.6-A.10).

\section{Results and Discussions}

\section{Surrogate Food Additives}

The 75\% DMSO/25\% methyl acetate solvent blend was first tested on HPLC to analyze surrogate food additive samples following a recommended gradient method [24]. Although the maximum pressure of this analysis was found to be 360 bar, higher than the highest operation pressure of 180 bar using acetonitrile as the organic mobile phase, it is still lower than the recommended instrument limitation of $400 \mathrm{bar}$, acceptable for typical operations. This high pressure is likely due to the high viscosity of the solvent blend caused by the presence of DMSO. When the solvent blend was used, the separation of the peaks was not satisfactory if the gradient was strictly followed. This is likely due to the reduced elution strength of the solvent blend. To resolve this issue, the gradient table was modified with an increased analysis time from 18 to $27 \mathrm{~min}$ to assist peak separation (Table A.6). The chromatogram obtained using the solvent blend (Fig. 2a) was compared to that obtained using acetonitrile (Fig. 2d). Note that finding the most optimal gradient for a new solvent blend was not the objective of this work and the method has the potential to be further optimized to reduce its operation time for each specific case.

When the $75 \%$ ethanol/25\% methyl acetate solvent blend was used as the organic mobile phase for food additive analysis on RP-HPLC, the operating pressure was found to be lower at 260 bar due to the absence of DMSO. The modified method for this blend is provided in Table A.7. When this solvent blend was used for analyzing surrogate food additives, the elution times of all the peaks was shorter (Fig. 2b), likely due to the fact that the mean HSP values for surrogate food additives (Table 2), particularly polar energy $(\delta \mathrm{P}=9.5)$ and hydrogen bonding energy $(\delta \mathrm{H}=14)$, are significantly closer to the corresponding values of the solvent blend $(\delta \mathrm{P}=8.4, \delta \mathrm{H}=16.5)$ compared to those of acetonitrile $(\delta \mathrm{P}=18.0, \delta \mathrm{H}=6.1)$.

When the $80 \%$ ethanol $/ 20 \%$ dimethyl carbonate solvent blend was used as the organic mobile phase for food additive analysis on RP-HPLC, the LC operation pressure was found to be at approximately 290 bar, between the operating pressures of the first and second blends. The modified method 
Fig. 2 RP-HPLC chromatograms using a the $75 \%$ DMSO/25\% methyl acetate blend, $\mathbf{b}$ the $75 \%$ ethanol $/ 25 \%$ methyl acetate blend, $\mathbf{c}$ the $80 \%$ ethanol $/ 20 \%$ dimethyl carbonate blend, and $\mathbf{d}$ acetonitrile as the organic mobile phase for the analysis of the surrogate food additives: (1) daidzin, (2) glycitin, (3) genistin, (4) daidzein, (5) glycitein, (6) genistein, and (7) apigenin

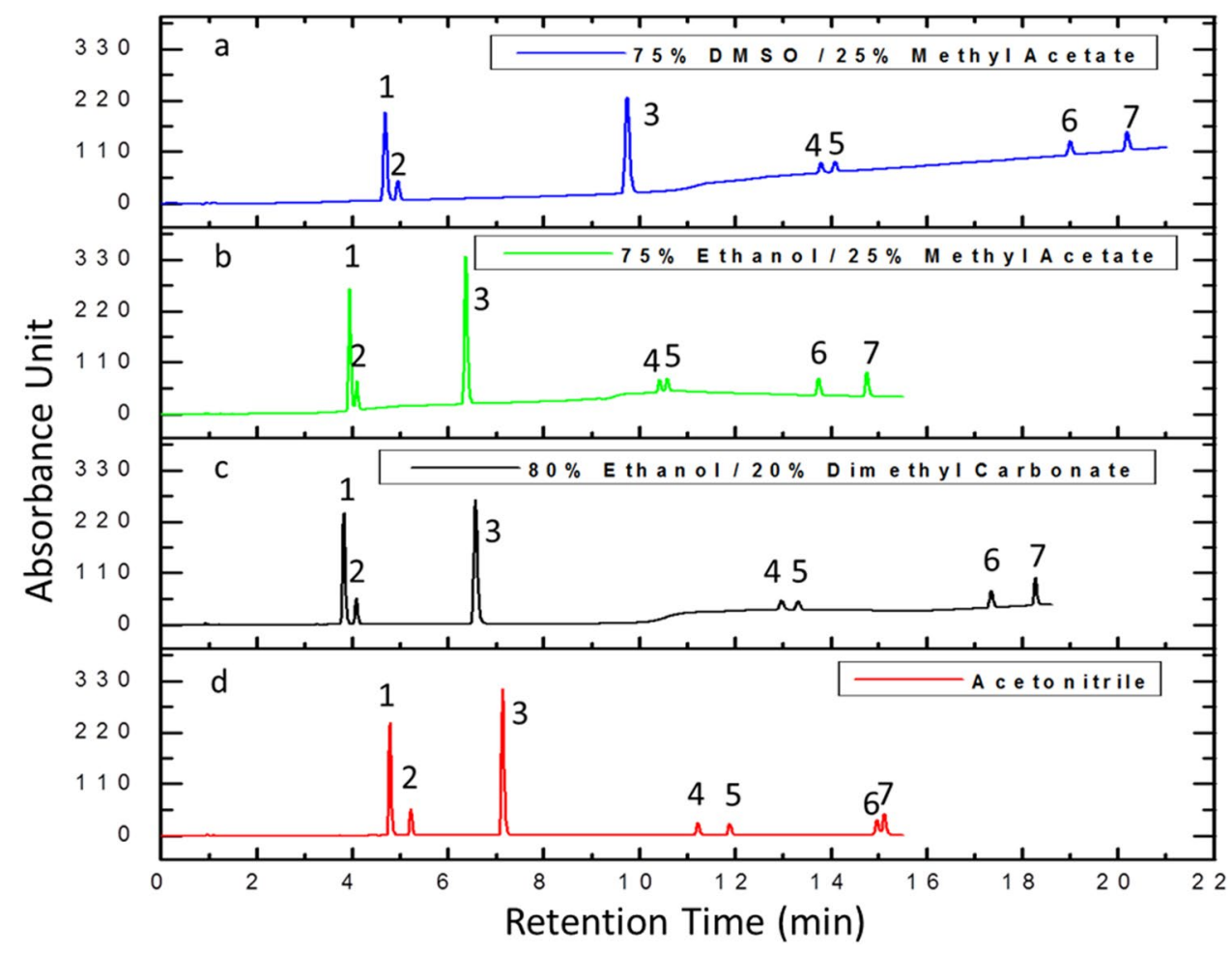

for the $80 \%$ ethanol $/ 20 \%$ dimethyl carbonate solvent blend is provided in Table A.8. Compared to acetonitrile, longer retention times of the peaks were observed with this solvent blend except the first three peaks (Fig. 2c).

Note that in the chromatograms shown in Fig. 2, baseline drifts were observed when safer solvent blends were used. This is due to the higher UV cutoffs of the solvents than that of acetonitrile (Fig. A.2). Particularly, both dimethyl sulfoxide (DMSO) and methyl acetate have the highest UV cutoffs among the solvents considered, at approximately $260 \mathrm{~nm}$ and $250 \mathrm{~nm}$, respectively (Fig. A.2). As a result, the blend of these two solvents resulted in the largest drift (Fig. 2a).

To assess the quality of the chromatograms when the solvent blends were used, the response factor of each peak, defined as integrated LC area per concentration of the species, using each of the three solvent blends was compared to that using acetonitrile (Fig. 3). In our work, the response factor of each compound was calibrated by its solutions in 4 different concentrations, prepared by following USP isoflavone method [38]. As shown in Fig. 3, the response factors obtained using all three solvent blends were very close to those obtained using acetonitrile. These comparable responses suggest that the solvent blends' higher UV absorbance and differences in elution strengths do not create significant issues of signal bias for the sets of analyte molecules examined in this work.

Peak width is another important parameter in evaluating the quality of the chromatograms. Narrower peaks allow better peak separation so that peak overlapping could be

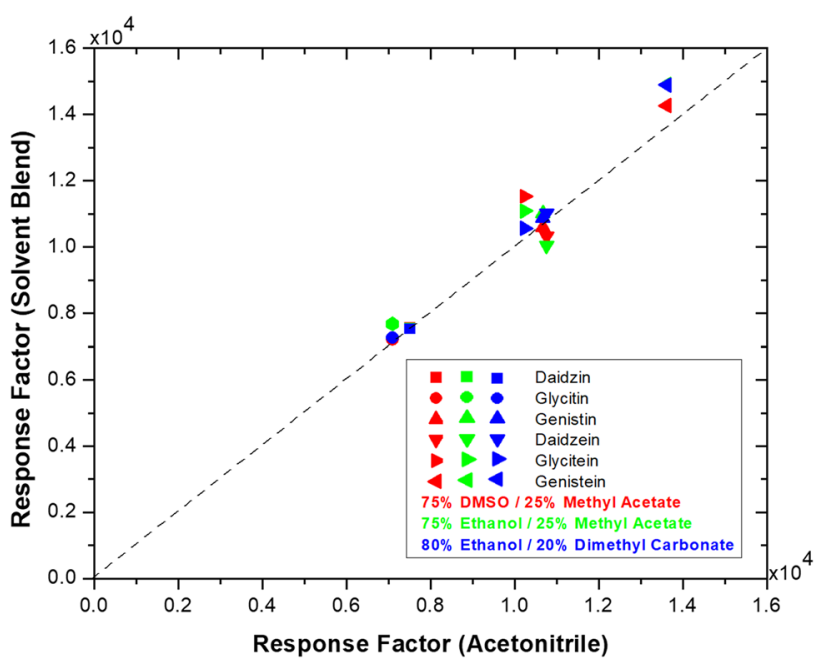

Fig. 3 Comparison of response factors of the surrogate food additives using acetonitrile and the candidate safer solvent blends as the organic mobile phase during LC operations

better avoided. However, the peak widths recorded under gradient conditions are strongly affected by the gradient profile. Typically, a faster gradient results in sharper peaks but at earlier elution times. To gain a more objective metric for peak width assessment, the column efficiency of each peak was calculated. Column efficiency measures the dispersion of a peak by calculating the number of theoretical plates per column, $N$, using the ratio of elution time and peak width as: 
$N=16\left(\frac{t_{r}}{W}\right)^{2}$

where $t_{r}$ is the elution time and $W$ is the peak width. The higher the number of theoretical plates, the sharper the peak is. Note that in our work, the reported values of the number of theoretical plates were calculated from peaks associated with the gradients that represent our best efforts to balance peak separation and peak shape. However, finding the most optimal gradient was not the main objective of this work. Consequently, there could still be a room to improve the methods for possible change of theoretical plates, although we expect the magnitude to be quite small.

Figure 4 depicts the ratio of the number of theoretical plates using the candidate safer solvent blends for each surrogate food additive peak to that using acetonitrile. Although peak areas (i.e., response factors) were very similar between the use of the safer solvent blends and acetonitrile, the peaks in general resulted in fewer theoretical plates when the solvent blends were used, particularly at higher analyte concentrations. The only exceptions are genistein and apigenin due to their longer elution times when the safer solvent blends were used (with similar peak widths, see Fig. A.3). The 75\% ethanol $/ 25 \%$ methyl acetate blend had the most consistent performance with the narrowest range of the ratio of the number of theoretical plates, in between 0.5 and 1 . The performances of the $80 \%$ ethanol $/ 20 \%$ dimethyl carbonate solvent blend and the $75 \%$ DMSO/25\% methyl acetate solvent blend were more inconsistent, having a broader range of the ratio of the number of theoretical plates in between 0.4 and 2.1 .

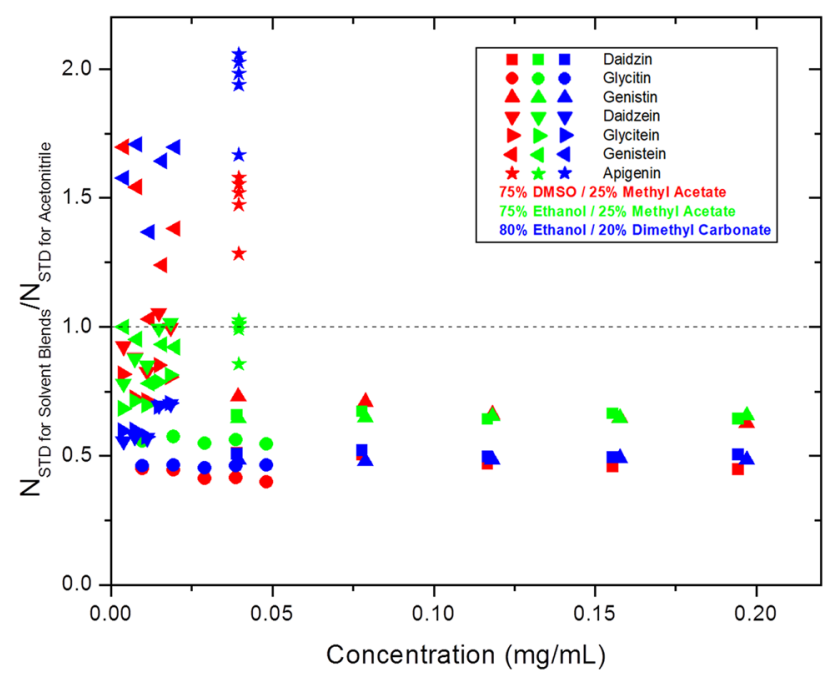

Fig. 4 Comparison of column efficiency, in terms of number of theoretical plates $(N)$, for the analysis of the surrogate food additives using the three different candidate safer solvent blends versus acetonitrile as the organic mobile phase during LC operations
Overall, our testing showed that $75 \%$ ethanol $/ 25 \%$ methyl acetate solvent blend has the lowest operating pressure among the three candidate solvent blends, with very similar response factors to those obtained from using acetonitrile during RP-HPLC analysis of the surrogate food additive molecules. The column efficiency in terms of the number of theoretical plates was also found to be acceptable. Furthermore, this solvent blend could potentially be more applicable for mass spectrometry (MS) usage since both components of the blend have low boiling points $\left(78.37{ }^{\circ} \mathrm{C}\right.$ for ethanol and $57.1^{\circ} \mathrm{C}$ for methyl acetate, respectively), as opposed to DMSO $\left(189^{\circ} \mathrm{C}\right)$ and dimethyl carbonate $\left(90^{\circ} \mathrm{C}\right)$. This finding is consistent with the recommendation by Tobiszewski et al. [21] that alcohols and esters can be considered as low environmental risk solvents. The use of ethanol and ethyl acetate was also suggested for, respectively, replacing tetrahydrofuran and acetonitrile as the mobile phase for LC applications [4, 7], although the blends of these solvents, which could provide new solvent properties, were never tested. While the use of ethanol in LC is not novel, the addition of methyl acetate, which has a lower viscosity and a lower cost than ethanol (see Table 1), is expected to mitigate the issues of pressure operation limitations and economic feasibility. To examine the robustness of this solvent blend, surrogate dye and wastewater pollutant analytes were further tested on RP-HPLC using this solvent blend as the organic mobile phase in place of acetonitrile.

\section{Surrogate Dye Molecules}

A group of surrogate dye molecules were tested using acetonitrile with a recommended method [25] and using the $75 \%$ ethanol $/ 25 \%$ methyl acetate blend with a modified method (Table A.9). The modified method was developed to observe all the peaks with acceptable peak separation for comparison. The resulting chromatograms (Fig. 5) show that there is a shift in the order of Peaks A and 6 when the 75\% ethanol $/ 25 \%$ methyl acetate solvent blend was used. In addition, all peaks have longer retention times except Peak 1.

The response factors of the surrogate dye molecules show that the $75 \%$ ethanol $/ 25 \%$ methyl acetate solvent blend gives very similar integrated areas to acetonitrile except impurity peak A (Fig. 6a), which does not pose a major concern. The comparison of the number of theoretical plates for the surrogate dye molecules using the $75 \%$ ethanol $/ 25 \%$ methyl acetate solvent blend to that using acetonitrile show that the solvent blend resulted in higher numbers of theoretical plates for most peaks than acetonitrile (Fig. 6b). This is due to the fact that the peaks from the analysis using the solvent blend have longer elution time (Fig. 6a) without a significant increase in peak widths, within 1.5 times compared to those obtained from acetonitrile (Fig. A.4). The only exception 
Fig. 5 RP-HPLC chromatograms using the $75 \%$ ethanol $/ 25 \%$ methyl acetate blend and acetonitrile as the organic mobile phase for the analysis of the surrogate dye molecules: (1) Blue 1, (2) Blue 3, (3) Blue 106, (4) Yellow 3, (5) Orange 3, (6) Red 1, (7) Blue 35, (8) Blue 124, and (9) Orange 37. Note that $\mathrm{A}$ is an impurity which is present regardless of solvents used

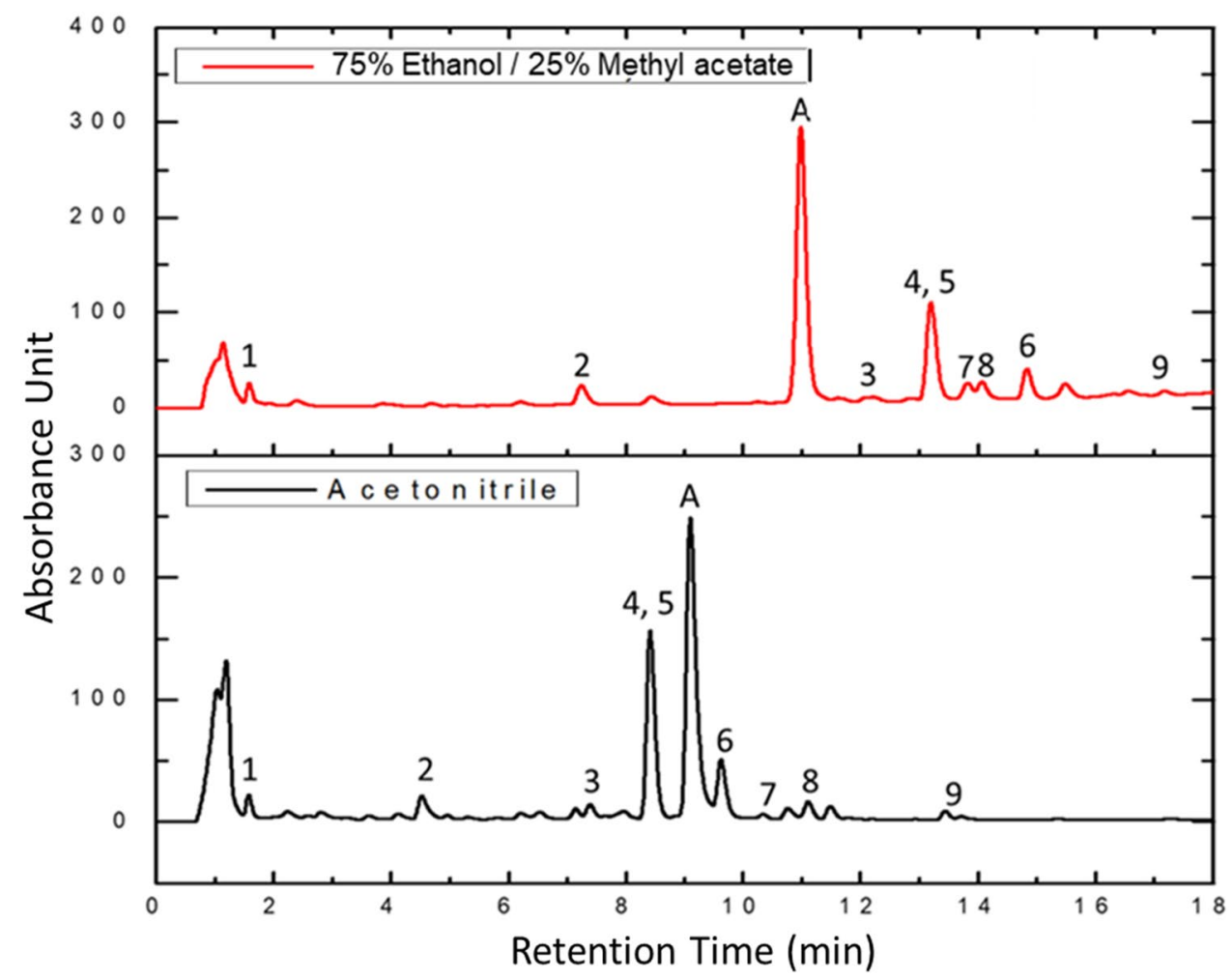

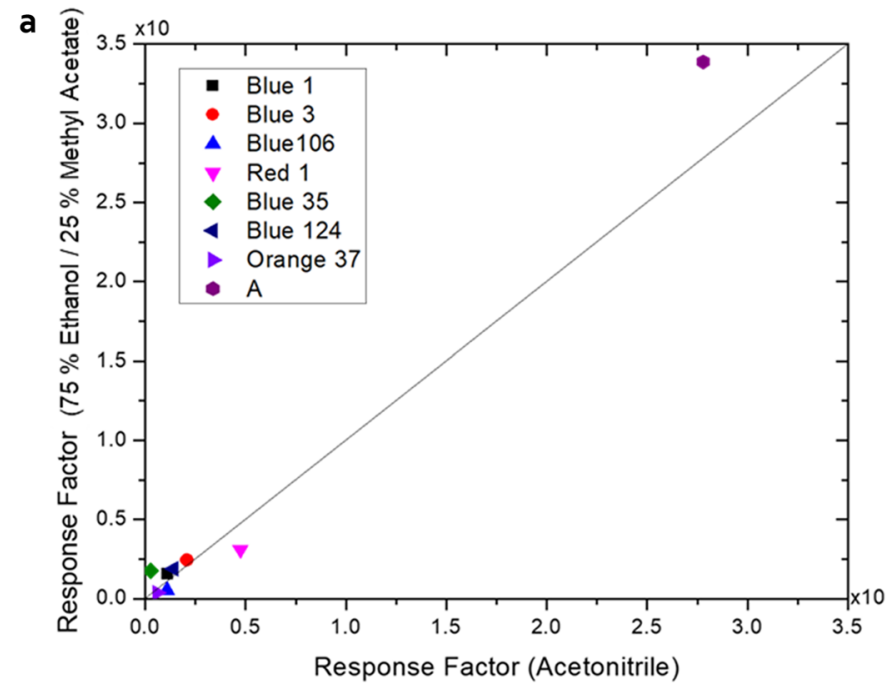

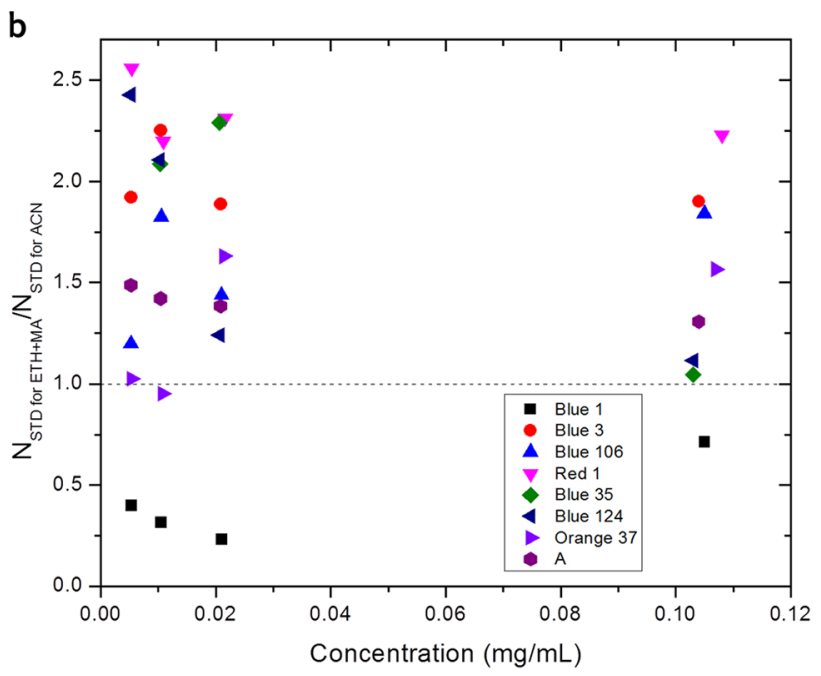

Fig. 6 Comparison of a response factors and $\mathbf{b}$ column efficiency, in terms of number of theoretical plates $(N)$, for the analysis of the surrogate dye molecules using the $75 \%$ ethanol/25\% methyl acetate solvent blend versus acetonitrile as the organic mobile phase during LC operations

was Blue 1 (Peak 1), which had a peak shape that presented difficulty in peak integration.

\section{Surrogate Wastewater Pollutants}

Surrogate wastewater pollutants for environmental analysis were also studied with the $75 \%$ ethanol $/ 25 \%$ methyl acetate solvent blend. The analytes were chosen based on an Agilent method [26] to detect trace organic compounds in wastewater. Instead of the Agilent column suggested in the method (Pursuit XRs C8, $100 \times 2.0 \mathrm{~mm}, 3 \mu \mathrm{m}$ ), a similar Waters column (Symmetry C8, $100 \times 2.1 \mathrm{~mm}, 3.5 \mu \mathrm{m}$ ) was used during the runs. The modified method developed for the $75 \%$ ethanol $/ 25 \%$ methyl acetate solvent blend is given in Table A.10. Since sucralose and TCPP do not show a significant UV absorbance (Fig. A.5), these two 
molecules could not be identified using a UV detector, regardless of solvents used. As a result, only three compounds (atenolol, sulfamethoxazole, and triclosan) were used for this analysis (Fig. 7). The chromatogram obtained from using the safer solvent blend produced peaks eluted with comparable amount of retention time. The peaks also had similar response factors to those obtained from using acetonitrile (Fig. 8a). Furthermore, the numbers of theoretical plates of the peaks were found to be acceptable, with no more than $\sim 2$ times lower than those using acetonitrile (Fig. 8b). Particularly, the atenolol peak was found to be approximately three times narrower than that
Fig. 7 RP-HPLC chromatograms using the $75 \%$ ethanol $/ 25 \%$ methyl acetate blend and acetonitrile as the organic mobile phase for analysis of the surrogate wastewater pollutants: (1) atenolol, (2) sulfamethoxazole, and (3) triclosan

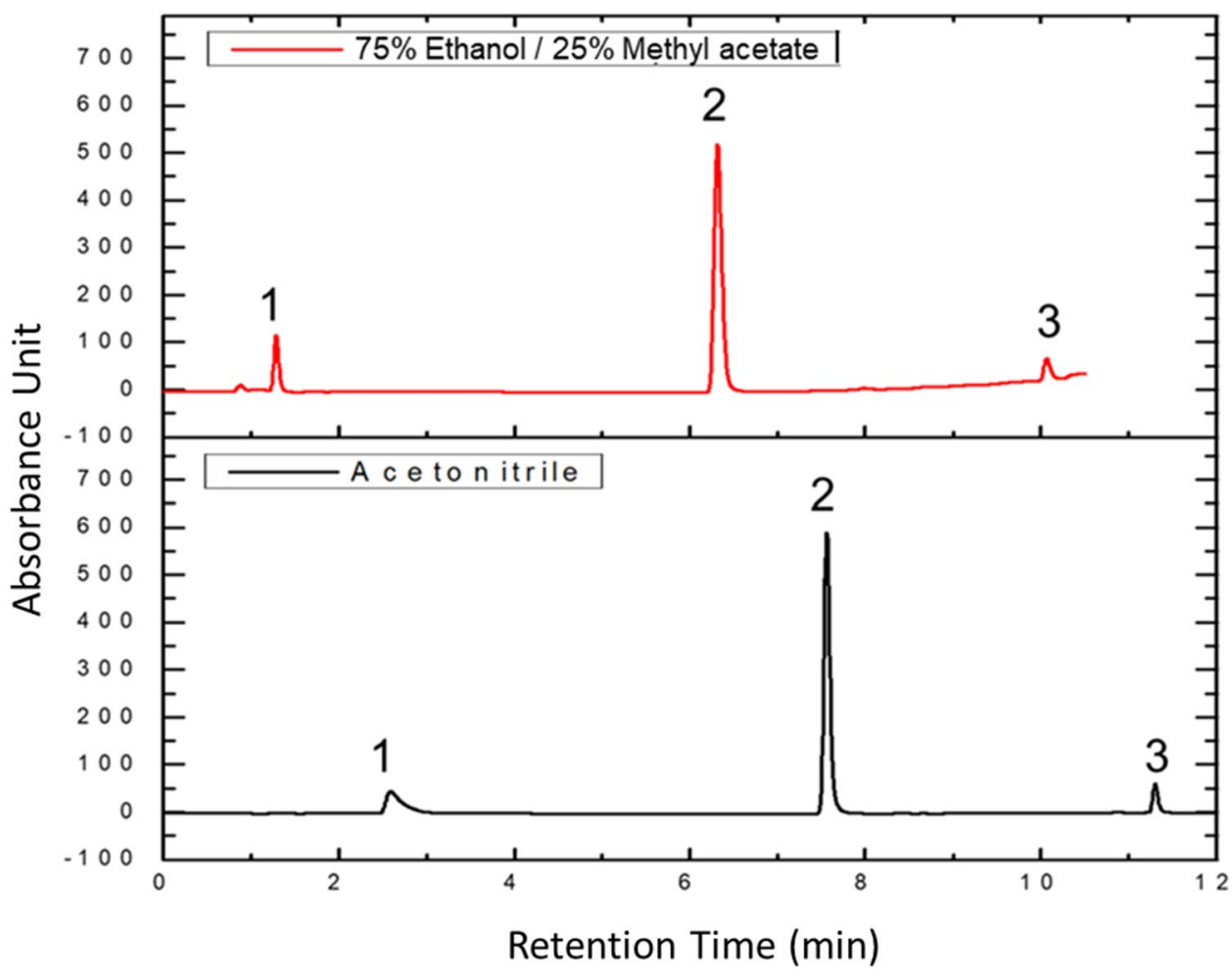

a

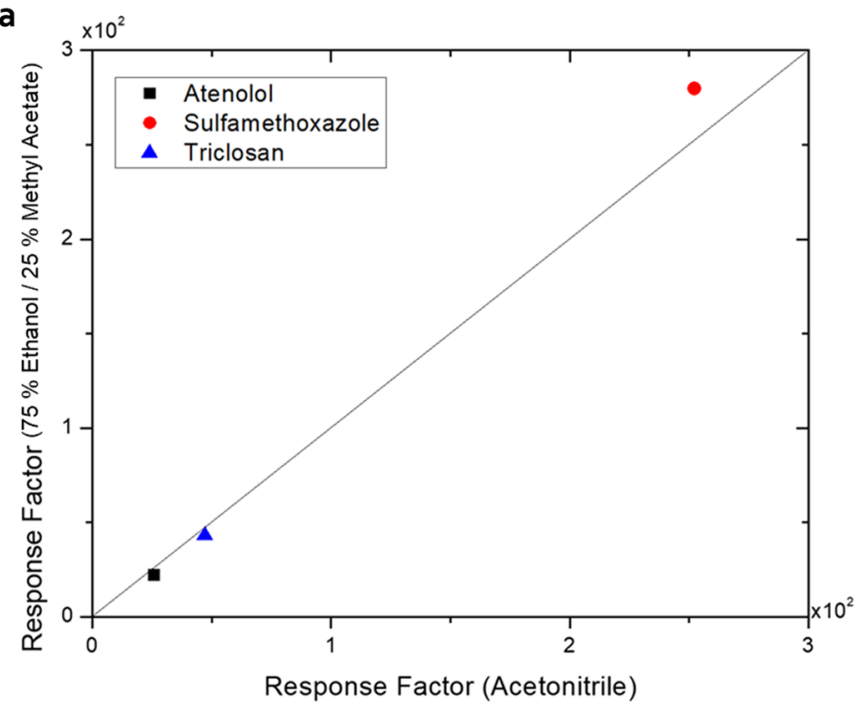

Fig. 8 Comparison of a response factor and b column efficiency, in terms of number of theoretical plates $(N)$, for the analysis of the surrogate wastewater pollutants using the $75 \%$ ethanol/25\% methyl b

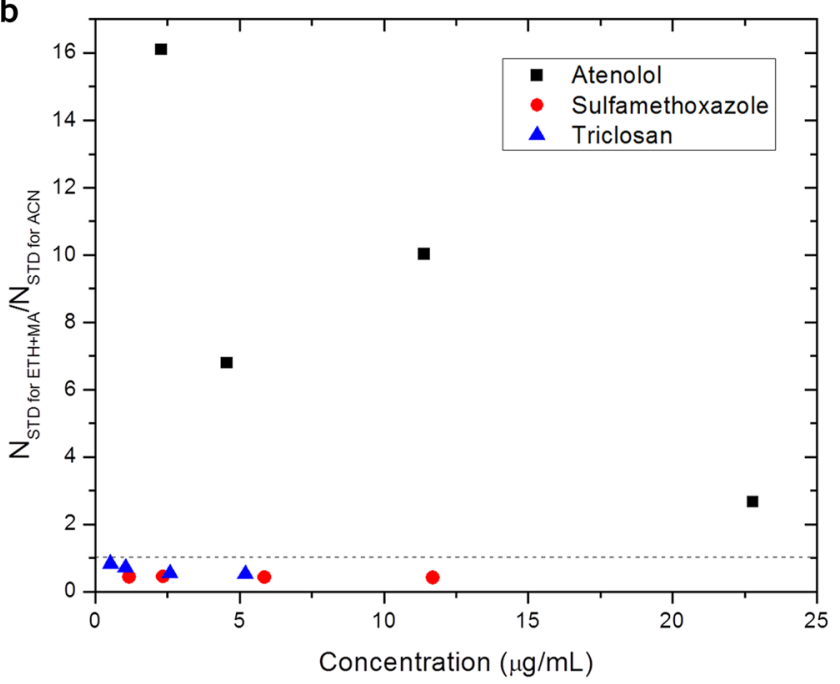

acetate solvent blend versus acetonitrile as the organic mobile phase during LC operations 
from using acetonitrile (Fig. A.6), resulting in high numbers of theoretical plates for the peak, especially at low concentrations.

\section{Conclusions}

A systematic approach for searching safer solvent blends to replace acetonitrile for LC applications was conducted. GreenScreen ${ }^{\circledR}$ for Safer Chemicals was used as the first filter to down-select solvent candidates safer than acetonitrile. A list of operation parameters was then employed to narrow down the candidates into five safer solvents as alternatives to acetonitrile, and HSPiP was used to determine the most probable compositions of solvent blends from these solvents for actual LC operations. It was found that a blend of $75 \%$ ethanol and $25 \%$ methyl acetate by volume provided the chromatograms with the best performance for RP-HPLC analysis of surrogate food, dye, and wastewater pollutant molecules, giving similar response factors and column efficiency compared to acetonitrile, with acceptable operation pressure. Our work shows that this safer solvent blend can address the safety concerns while keeping its performance as close to that of acetonitrile as possible, despite some known compromises in wavelength cutoff and viscosity. The acceptable performance for the three sets of surrogate molecules in very different application fields also suggests that our solvent blend, not intended to totally replace acetonitrile, can still find its areas of applications, helping mitigate the environmental, health, and safety concerns caused by acetonitrile.

Although this solvent blend was developed based on $\mathrm{UV}-\mathrm{Vis}$ detectors, we are confident that the success is transferrable to mass spectrometry (MS) detections. Liquid chromatography equipped with mass spectrometers (LC/ MS) is widely used in compound identification or quantification of molecules in low concentrations where typical UV-Vis or optical detectors do not have the sensitivity. Electrospray ionization is the most commonly used ionization mode for LC/MS applications, and the volatility of the mobile phase (i.e., solvent) is the most critical factor to the ionization efficiencies of the analytes. A solvent that does not evaporate well will produce ionized droplets that are too large to provide sufficient signals. Both components of our recommended safer solvent blend (i.e., 75\% ethanol and 25\% methyl acetate by volume), however, have boiling points (i.e., $78.4{ }^{\circ} \mathrm{C}$ for ethanol and $57.1{ }^{\circ} \mathrm{C}$ for methyl acetate) comparable to the solvents commonly used in HPLC (such as acetonitrile at $82{ }^{\circ} \mathrm{C}$ and methanol at $64.7^{\circ} \mathrm{C}$ ), suggesting good compatibility with MS applications. We will examine this compatibility as part of our future studies to test the robustness of the suggested solvent blend for acetonitrile replacement.
Supplementary Information The online version contains supplementary material available at https://doi.org/10.1007/s10337-021-04061-8.

Acknowledgements This research was funded by the Massachusetts Toxics Use Reduction Institute, and technical input was provided by Waters Corporation.

\section{Declarations}

Conflict of Interest The authors declare that they have no known competing financial interests or personal relationships that could have appeared to influence the work reported in this paper.

Ethical Approval This article does not contain any studies with human participants or animals performed by any of the authors.

Open Access This article is licensed under a Creative Commons Attribution 4.0 International License, which permits use, sharing, adaptation, distribution and reproduction in any medium or format, as long as you give appropriate credit to the original author(s) and the source, provide a link to the Creative Commons licence, and indicate if changes were made. The images or other third party material in this article are included in the article's Creative Commons licence, unless indicated otherwise in a credit line to the material. If material is not included in the article's Creative Commons licence and your intended use is not permitted by statutory regulation or exceeds the permitted use, you will need to obtain permission directly from the copyright holder. To view a copy of this licence, visit http://creativecommons.org/licenses/by/4.0/.

\section{References}

1. Gaber Y, Törnvall U, Kumar M, Amin MA, Hatti-Kaul R (2011) HPLC-EAT (environmental assessment tool): a tool for profiling safety, health and environmental impacts of liquid chromatography methods. Green Chem 13:2021-2025

2. Snyder LR, Kirkland JJ, Dolan JW (2009) Introduction to modern liquid chromatography. Wiley \& Sons Inc., Hoboken

3. Sheldon RA (2012) Fundamentals of green chemistry: efficiency in reaction design. Chem Soc Rev 41:1437-1451

4. Shen Y, Chen B, Van Beek TA (2015) Alternative solvents can make preparative liquid chromatography greener. Green Chem 17:4073-4081

5. Anastas PT, Kirchhoff MM (2002) Origins, current status, and future challenges of green chemistry. Acc Chem Res 35:686-694

6. Tobiszewski M, Mechlińska A, Namieśnik J (2010) Green analytical chemistry - theory and practice. Chem Soc Rev 39:2869-2878

7. Yabré M, Ferey L, Somé IT, Gaudin K (2018) Greening reversedphase liquid chromatography methods using alternative solvents for pharmaceutical analysis. Molecules 23:1065

8. Tobiszewski M, Namieśnik J (2017) Greener organic solvents in analytical chemistry. Curr Opin Green Sustain Chem 5:1-4

9. Koel M (2016) Do we need green analytical chemistry? Green Chem 18:923-931

10. Snyder L (1978) Classification of the solvent properties of common liquids. J Chromatogr Sci 16:223-234

11. Johnson AR, Vitha MF (2011) Chromatographic selectivity triangles. J Chromatogr A 1218:556-586

12. Jimenez-Gonzalez C, Curzons AD, Constable DJ, Cunningham VL (2004) Expanding GSK's solvent selection guide-application of life cycle assessment to enhance solvent selections. Clean Technol Environ Policy 7:42-50 
13. Alfonsi K, Colberg J, Dunn PJ, Fevig T, Jennings S, Johnson TA, Kleine HP, Knight C, Nagy MA, Perry DA (2008) Green chemistry tools to influence a medicinal chemistry and research chemistry based organisation. Green Chem 10:31-36

14. Prat D, Pardigon O, Flemming H-W, Letestu S, Ducandas V, Isnard P, Guntrum E, Senac T, Ruisseau S, Cruciani P, Hosek P (2013) Sanofi's solvent selection guide: a step toward more sustainable processes. Org Process Res Dev 17:1517-1525

15. Henderson RK, Jiménez-González C, Constable DJ, Sarah RA, Inglis GG, Fisher G, Sherwood J, Binks SP, Curzons AD (2011) Expanding GSK's solvent selection guide-embedding sustainability into solvent selection starting at medicinal chemistry. Green Chem 13:854-862

16. Hargreaves CR (2008) Collaboration to deliver a solvent selection guide for the pharmaceutical industry. In 2008 AIChE Annual Meeting, Philadelphia, PA

17. Prat D, Hayler J, Wells A (2014) A survey of solvent selection guides. Green Chem 16:4546-4551

18. Alder CM, Hayler JD, Henderson RK, Redman AM, Shukla L, Shuster LE, Sneddon HF (2016) Updating and further expanding GSK's solvent sustainability guide. Green Chem 18:3879-3890

19. Prat D, Wells A, Hayler J, Sneddon H, McElroy CR, Abou-Shehada S, Dunn PJ (2016) CHEM21 selection guide of classical- and less classical-solvents. Green Chem 18:288-296

20. Tobiszewski M, Tsakovski S, Simeonov V, Namieśnik J, PenaPereira F (2015) A solvent selection guide based on chemometrics and multicriteria decision analysis. Green Chem 17:4773-4785

21. Tobiszewski M, Namieśnik J, Pena-Pereira F (2017) Environmental risk-based ranking of solvents using the combination of a multimedia model and multi-criteria decision analysis. Green Chem 19:1034-1042

22. Barry CP, Morose GJ, Begin K, Atwater M, Hansen CJ (2017) The identification and screening of lower toxicity solvents for contact adhesives. Int J Adhes Adhes 78:174-181

23. Hansen CM (2002) Hansen solubility parameters: a user's handbook. CRC Press, Boca Raton

24. Yang J, Benvenuti M, Cleland G (2017) Determination of isoflavones in dietary supplements: a comparison of mass detection with UV detection. Waters Corporation

25. Twohig M, O'Leary M, Cooper J (2016) Analysis of disperse dyes using the ACQUITY arc system with PDA and mass detection, and empower software. Waters Corporation
26. Wu S, dos Santos MM, Daniels K, Snyder S, Anumol T (2015) Identification of a panel of 20 indicator compounds in wastewater effluent using rapid, direct injection LC/MS/MS on the Agilent 6490 triple quadrupole LC/MS. Agilent Technologies

27. Clean Production Action (2018) GreenScreen ${ }^{\circledR}$ for Safer Chemicals - Hazard Assessment Guidance v1.4; Clean Production Action

28. Clean Production Action GreenScreen List Translator ${ }^{\mathrm{TM}}$ : A listbased hazard screening approach. https://www.greenscreenchem icals.org/learn/greenscreen-list-translator

29. Molbase http://www.molbase.com/

30. Morose G, Marshall J, McCarthy A, Harripersaud V, Giarrosso A (2017) Assessment of safer and effective alternatives to methylene chloride for paint stripping products. Massachusetts Toxics Use Reduction Institute (TURI)

31. Snyder LR, Kirkland J, Glajch JL (1997) Practical HPLC method development. John Willey \& Sons Inc., New York

32. National Library of Medicine PubChem: an open chemistry database at the National Institutes of Health. https://pubchem.ncbi. nlm.nih.gov/.

33. European Chemicals Agency Information on Chemicals. https:// www.echa.europa.eu/.

34. Chemical Book. https://www.chemicalbook.com/.

35. Hansen CM (1967) The three dimensional solubility parameter and solvent diffusion coefficient. Danish Technical Press, Copenhagen

36. Abraham MH, Ibrahim A, Zissimos AM (2004) Determination of sets of solute descriptors from chromatographic measurements. J Chromatogr A 1037:29

37. Abbott $S$ (2021) Solubility science: principles and practice. Steven Abbott TCNF Ltd., Ipswich

38. U.S. Pharmacopeia National Formulary (2016) USP 39 NF 34 The United States Pharmacopeia - National Formulary (USP-NF)

Publisher's Note Springer Nature remains neutral with regard to jurisdictional claims in published maps and institutional affiliations. 